\title{
Formation of silicon monoxide by radiative association: the impact of resonances
}

\author{
Robert C Forrey ${ }^{1} \dagger$, James F Babb ${ }^{2}$, Phillip C Stancil ${ }^{3}$ and \\ Brendan M McLaughlin $2,4 \ddagger$ \\ ${ }^{1}$ Department of Physics, Pennsylvania State University, Berks Campus, \\ Reading, PA 19610-6009, USA \\ ${ }^{2}$ Institute for Theoretical Atomic, Molecular, and Optical Physics (ITAMP), \\ Harvard-Smithsonian Center for Astrophysics, MS-14, Cambridge, MA 02138, \\ USA \\ ${ }^{3}$ Department of Physics and Astronomy and the Center for Simulational Physics, \\ University of Georgia, Athens, GA 30602-2451, USA \\ ${ }^{4}$ Centre for Theoretical Atomic, Molecular and Optical Physics (CTAMOP), \\ School of Mathematics and Physics, The David Bates Building, 7 College Park, \\ Queen's University Belfast, Belfast BT7 1NN, UK
}

\begin{abstract}
Detailed quantum chemistry calculations within the multireference configuration interaction approximation with the Davidson correction (MRCI+Q) are presented using an aug-cc-pV6Z basis set, for the potential energy curves and transition dipole moments between low lying molecular states of singlet spin symmetry for the $\mathrm{SiO}$ molecule. The high quality molecular data are used to obtain radiative association cross sections and rate coefficients for collisions between ground state $\mathrm{Si}$ and $\mathrm{O}$ atoms. Quantal calculations are compared with semiclassical results. Using a quantum kinetic theory of radiative association in which quasibound levels are assumed to be in local thermodynamic equilibrium, we find that resonances play an important role in enhancing the rate coefficients at low temperatures by several orders of magnitude from that predicted by standard quantum scattering formulations. These new molecular formation rates may have important implications for applications in astrophysics.

PACS numbers: 32.80.Fb, 31.15.Ar, 32.80.Hd, and 32.70.-n
\end{abstract}

Short title: Formation of $\mathrm{SiO}$ by radiative association J. Phys. B: At. Mol. Opt. Phys. : 12 September 2018

$\dagger$ Corresponding author, E-mail: rcf6@psu.edu

$\ddagger$ Corresponding author, E-mail: bmclaughlin899@btinternet.com 


\section{Introduction}

The formation of molecules through radiative association can be an important astrophysical process contributing to chemical evolution in environments that are free of dust and hydrogen. In particular, $\mathrm{CO}$ and $\mathrm{SiO}$ were detected spectrally in the ejecta of Type II supernovae (SNe) between about 100 days and 500 days from the initial explosions. The first molecular detection was from SN1987A, and theoretical studies indicated that the primary mechanism leading to formation of $\mathrm{CO}$ and $\mathrm{SiO}$ is radiative association [1-3]. $\mathrm{CO}$ and/or $\mathrm{SiO}$ has been detected in several subsequent supernovae [4]. The radiative association of $\mathrm{C}$ and $\mathrm{O}$ has been investigated in some detail [5-7]. It is well known that $\mathrm{CO}$ and $\mathrm{SiO}$ play fundamental roles in the dust formation process, though SN1987A remains the best-studied event due to its relative proximity. The formation of $\mathrm{SiO}$ is thought to be a key step in the subsequent formation of silicates and dust (e.g. [8]).

The rate coefficient for the radiative association of $\mathrm{Si}$ and $\mathrm{O}$,

$$
\mathrm{Si}+\mathrm{O} \rightarrow \mathrm{SiO}+\mathrm{h} \nu,
$$

where $h \nu$ represents the emitted photon, was calculated by Andreazza, Marinho, and Singh [9] using a semiclassical theory with empirical molecular data. In this paper, we investigate the radiative association of ground state $\mathrm{Si}$ and $\mathrm{O}$ using accurate molecular data and several theoretical approaches for the calculation of radiative association cross sections. A semiclassical approach to calculating the rate coefficient [10] is appropriate for heavy atoms such as $\mathrm{Si}$ and $\mathrm{O}$ and may provide a suitable estimate of the non-resonant contribution for temperatures as low as several hundred degrees Kelvin (K). It does not, however, without additional treatment, take into account resonances that can occur for certain values of the collision energy. Standard quantum-mechanical methods for calculating the cross sections are also available, which intrinsically include resonance effects. These methods generally add radiative broadening in order to exclude very narrow resonances from contributing to the rate constant [7,11-13]. Recently, a quantum kinetic theory [14] showed that the narrow resonances should not be neglected and predicted they would generally lead to large enhancements in molecular formation rates. Here, we investigate this possibility for $\mathrm{Si}$ and $\mathrm{O}$ collisions for the conditions of local thermodynamic equilibrium (LTE).

\section{Molecular Structure}

In the present study the potential energy curves and transition dipole matrix elements of the low lying electronic states are calculated by using a state-averaged-multiconfiguration-self-consistent-field (SA-MCSCF) approach, followed by multi-reference configuration interaction (MRCI) calculations together with the Davidson correction $(\mathrm{MRCI}+\mathrm{Q})$ [15]. The SA-MCSCF method is used as the reference wavefunction for the MRCI calculations in all our work. Low lying singlet electronic states and the transition dipole matrix elements connecting these states are calculated and used in subsequent dynamical calculations for the radiative association process. Potential energy curves (PECs) and transition dipole moments (TDMs) as a function of internuclear distance $R$ are computed out to a bond separation of $R=20$ Bohr. The basis sets used in our work are the augmented correlation consistent polarized aug-cc-pV6Z (AV6Z) basis sets [16-18]. All the PEC and TDM calculations were performed with the quantum chemistry MOLPRO 2012.1.21 program package [19] 


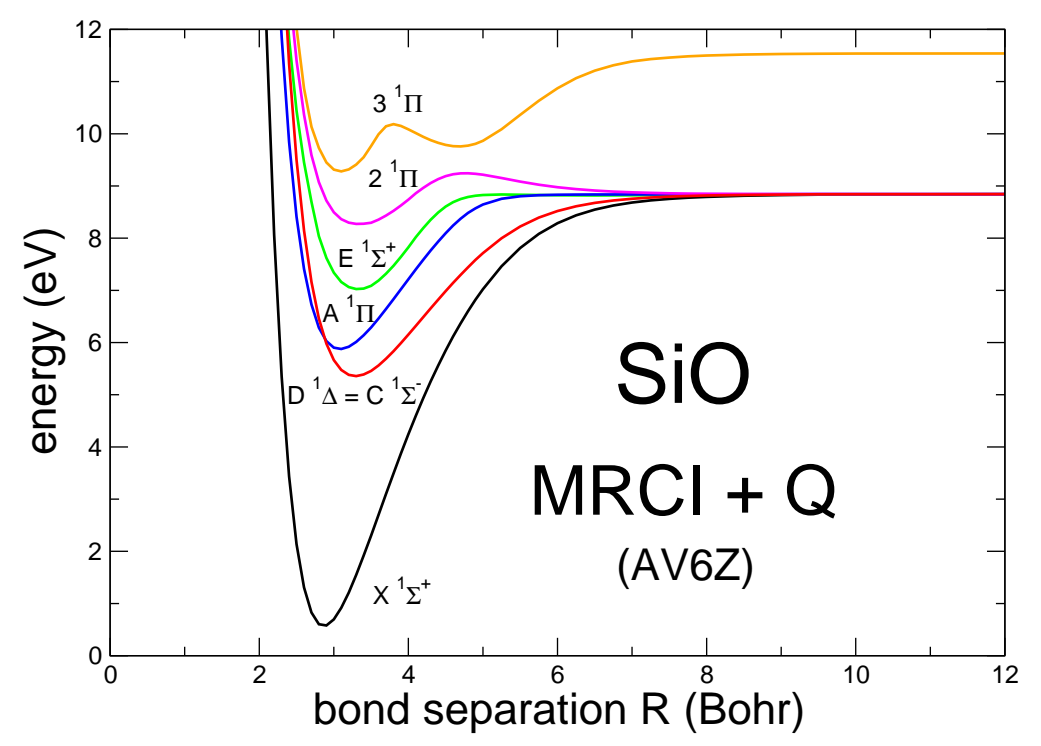

Figure 1. Relative electronic energies $(\mathrm{eV})$ for the $\mathrm{SiO}$ molecule as a function of bond separation at the MRCI+Q level of approximation with an AV6Z basis. The $\mathrm{X}^{1} \Sigma^{+}$ground state along with several of the low lying states, for the singlet symmetries; $\mathrm{C}^{1} \Sigma^{-}, \mathrm{E}^{1} \Sigma^{+}, \mathrm{A}^{1} \Pi, 2^{1} \Pi$ and $3^{1} \Pi$ are illustrated.

running on parallel architectures. The computations were performed in the Abelian subgroup $\mathrm{C}_{2 v}$ with the order of irreducible representations being $\left(A_{1}, B_{1}, B_{2}, A_{2}\right)$. We note that the full natural Abelian symmetry group for a diatomic molecule like $\mathrm{SiO}$ is $\mathrm{C}_{\infty v v}$ symmetry. When symmetry is reduced from $\mathrm{C}_{\infty v}$ to $\mathrm{C}_{2 v}$, the correlating relationships are $\sigma \rightarrow a_{1}, \pi \rightarrow\left(b_{1}, b_{2}\right), \delta \rightarrow\left(a_{1}, a_{2}\right)$. In order to take account of shortrange interactions we employed the non-relativistic state-averaged complete activespace-self-consistent- field (SA-CASSCF)/MRCI method $[20,21]$ available within the MOLPRO [19] ab initio quantum chemistry suite of codes. In detail, for the $\mathrm{SiO}$ molecule, eight molecular orbitals (MOs) are put into the active space, including four $a_{1}$, two $b_{1}$ and two $b_{2}$ symmetry MOs which correspond to the $3 s 3 p$ shell of Si and $2 s 2 p$ shell of $\mathrm{O}$ atoms. The rest of the electrons in the $\mathrm{SiO}$ molecule are put into the closedshell orbitals, including four $a_{1}$, one $b_{1}$ and one $b_{2}$ symmetry MOs. The molecular orbitals for the MRCI procedure are obtained from the SA-MCSCF method, where the averaging process was carried out on the lowest three ${ }^{1} \Sigma\left({ }^{1} A_{1}\right)$, three ${ }^{1} \Pi\left({ }^{1} B_{1}\right)$, and one ${ }^{1} \Delta\left({ }^{1} A_{2}\right)$ molecular states of this molecule (note: the ${ }^{1} \Delta$ and ${ }^{1} \Sigma^{-}$states are degenerate). We use these fourteen MOs $\left(8 a_{1}, 3 b_{1}, 3 b_{2}, 0 a_{2}\right)$, i.e. $(8,3,3,0)$, to perform all the PEC calculations of these electronic states in the $\mathrm{MRCI}+\mathrm{Q}$ approximation. Fig 1 shows the low lying singlet states for $\mathrm{SiO}$ to their respective dissociation limits. 


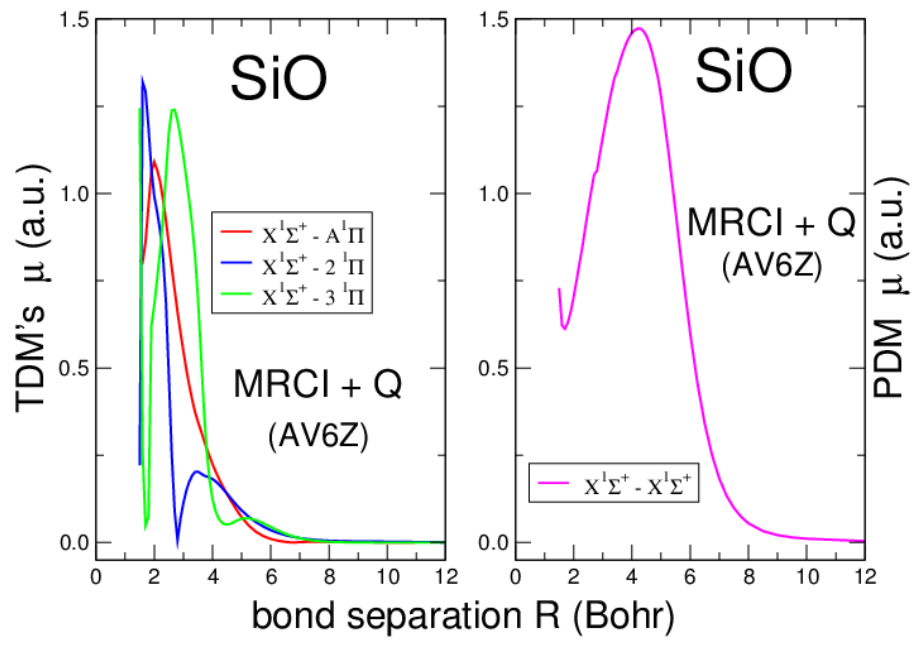

Figure 2. Absolute magnitude of the $\mathrm{SiO}$ transition dipole moments (TDMs), from the ground state, the left panel is $\mu(R)$ for transitions to the low lying singlet $\Pi$ states namely; $\mathrm{X}^{1} \Sigma^{+} \rightarrow A^{1} \Pi, 2^{1} \Pi$, and $3^{1} \Pi$. The right panel is the absolute magnitude of the permanent dipole moment $(\mathrm{PDM})$ for the $\mathrm{X}^{1} \Sigma^{+}$ground state of $\mathrm{SiO}$. The MRCI $+\mathrm{Q}$ approximation is used with an AV6Z basis set within the MOLPRO suite of codes to determine all the moments as a function of the internuclear distance $R$.

In order to accurately determine the PECs, the point spacing intervals used here is $0.1 \mathrm{a}_{0}(0.052918 \AA)$ for each electronic state, out to $5 \mathrm{a}_{0}$ except near the equilibrium internuclear separation where the spacing is $0.01 \mathrm{a}_{0}$. Here, a smaller step size is adopted around the equilibrium position of each electronic state so that the properties of each PEC can be displayed more clearly. For the present internuclear distances from 1.5 to $5 \mathrm{a}_{0}$, the PEC of each electronic state obtained is smooth and convergent. Beyond a bond separation of $5 \mathrm{a}_{0}$ a spacing of $0.25 \mathrm{a}_{0}$ was used. It is clearly seen that the two atoms, $\mathrm{Si}$ and $\mathrm{O}$, are completely separated beyond about $10 \mathrm{a}_{0}$. In Fig 2 we present the dipole moments as a function of $R$. The left panel of Fig 2 illustrates the absolute magnitude of the TDMs connecting the low lying singlet $\Pi$ states to the ground state. The right panel of Fig 2 shows the absolute magnitude of the permanent dipole moment (PDM) for the $\mathrm{X}^{1} \Sigma^{+}$ground state as a function of $R$. We note as the molecule dissociates, both the TDMs and the PDM are smooth functions of $R$. The calculated potential energies as a function of $R$ show excellent agreement with previous relativistic-core-potential (RECP) based multi-reference single and double CI (MRDCI) calculations [22], MRCI $+\mathrm{Q}$ calculations [23] and results from the EXOMOL project [24].

For the radiative association process we are interested in the $\mathrm{X}^{1} \Sigma^{+}$and the $\mathrm{A}^{1} \Pi$ molecular states of $\mathrm{SiO}$ and the transition dipole moment $\mu_{X \rightarrow A}(R)$ connecting these states as a function of bond length. The present $\mathrm{MRCI}+\mathrm{Q}$ values for the $\mathrm{X}-\mathrm{A}$ transition dipole moment as a function of bond length are in excellent agreement with the earlier self-consistent field plus configuration interaction $(\mathrm{SCF}+\mathrm{CI})$ calculations 
Table 1. Spectroscopic constants for the $\mathrm{X}^{1} \Sigma^{+}$and the $\mathrm{A}^{1} \Pi$ states of the $\mathrm{SiO}$ molecule. The equilibrium bond distance $r_{e}$ and the dissociation energies $D_{e}$ are presented. The present MRCI $+\mathrm{Q}$ results shown are compared with previous theoretical work and with experiments.

\begin{tabular}{|c|c|c|c|c|}
\hline Basis & State & Method & $r_{e}(\AA)$ & $D_{e}(\mathrm{eV})$ \\
\hline $\begin{array}{l}\text { aug-cc-pV6Z } \\
\text { aug-cc-pV5Z } \\
\text { aug-cc-pV6Z } \\
\text { aug-cc-pV6Z } \\
\text { CBS }\end{array}$ & $\mathrm{X}^{1} \Sigma^{+}$ & $\begin{array}{c}\mathrm{MRCI}+\mathrm{Q}^{a} \\
\mathrm{MRCI}+\mathrm{Q}^{b} \\
\mathrm{MRCI}+\mathrm{Q}^{b} \\
\mathrm{MRCI}+\mathrm{Q} / \mathrm{CV}+\mathrm{DK}{ }^{b} \\
\mathrm{MRCI}+\mathrm{Q} / \mathrm{CV}+\mathrm{DK}+56^{b}\end{array}$ & $\begin{array}{l}1.5153 \\
1.5169 \\
1.5159 \\
1.5114 \\
1.5100\end{array}$ & $\begin{array}{l}8.2748 \\
8.2443 \\
8.2800 \\
8.3281 \\
8.3776\end{array}$ \\
\hline $\begin{array}{l}\mathrm{Si}(8 \mathrm{~s} / 7 \mathrm{p} / 4 \mathrm{~d} / 3 \mathrm{f} / 2 \mathrm{~g} / 1 \mathrm{~h}) \\
\mathrm{O}(7 \mathrm{~s} / 6 \mathrm{p} / 4 \mathrm{~d} / 3 \mathrm{f} / 2 \mathrm{f} / 1 \mathrm{~h})\end{array}$ & & $\mathrm{CCSDT}^{c}$ & 1.5156 & 8.2482 \\
\hline $\begin{array}{l}\text { STO basis } \\
\text { STO basis }\end{array}$ & & $\begin{array}{c}\mathrm{SCF}+\mathrm{CI}^{d} \\
\mathrm{MCSCF}+\mathrm{CI}^{d}\end{array}$ & $\begin{array}{c}1.4960 \\
-\end{array}$ & $\begin{array}{l}8.1000 \\
8.1500\end{array}$ \\
\hline cc-pVTZ/D-Gauss-Xfit & & $\begin{array}{c}\mathrm{CASSCF} / \mathrm{MRCI}^{e} \\
\mathrm{RSPT}^{f}\end{array}$ & $\begin{array}{l}1.5237 \\
1.5328\end{array}$ & \\
\hline $\begin{array}{l}\text { Si }(7 \mathrm{~s} / 6 \mathrm{p} / 4 \mathrm{~d} / 1 \mathrm{f}) \\
\mathrm{O}(4 \mathrm{~s} / 4 \mathrm{p} / 1 \mathrm{~d})\end{array}$ & & $\mathrm{RECP}+\mathrm{MRDCI}^{g}$ & 1.5210 & 7.8700 \\
\hline Experiment & & & $1.5097^{h}$ & $\begin{array}{c}8.3368^{h} \\
8.26 \pm 0.13^{i} \\
8.36 \pm 0.09^{j} \\
8.18 \pm 0.30^{k}\end{array}$ \\
\hline $\begin{array}{l}\text { aug-cc-pV6Z } \\
\text { aug-cc-pV5Z } \\
\text { aug-cc-pV6Z } \\
\text { aug-cc-pV6Z } \\
\text { CBS }\end{array}$ & $\mathrm{A}^{1} \Pi$ & $\begin{array}{c}\mathrm{MRCI}+\mathrm{Q}^{a} \\
\mathrm{MRCI}+\mathrm{Q}^{b} \\
\mathrm{MRCI}+\mathrm{Q}^{b} \\
\mathrm{MRCI}+\mathrm{Q} / \mathrm{CV}+\mathrm{DK}{ }^{b} \\
\mathrm{MRCI}+\mathrm{Q} / \mathrm{CV}+\mathrm{DK}+56^{b}\end{array}$ & $\begin{array}{l}1.6315 \\
1.6309 \\
1.6295 \\
1.6249 \\
1.6229\end{array}$ & $\begin{array}{l}2.9693 \\
2.9662 \\
2.9926 \\
3.0146 \\
3.0510\end{array}$ \\
\hline STO basis & & $\mathrm{SCF}+\mathrm{CI}^{d}$ & 1.4960 & 2.44 \\
\hline cc-pVTZ/D-Gauss-Xfit & & $\begin{array}{c}\mathrm{CASSCF} / \mathrm{MRCI}^{e} \\
\mathrm{RSPT}^{f}\end{array}$ & $\begin{array}{l}1.7599 \\
1.8160\end{array}$ & \\
\hline $\begin{array}{l}\text { Si }(7 \mathrm{~s} / 6 \mathrm{p} / 4 \mathrm{~d} / 1 \mathrm{f}) \\
\mathrm{O}(4 \mathrm{~s} / 4 \mathrm{p} / 1 \mathrm{~d})\end{array}$ & & $\mathrm{RECP}+\mathrm{MRDCI}^{g}$ & 1.6500 & 2.5600 \\
\hline Experiment & & & $1.6206^{h}$ & $\begin{array}{c}3.0259^{h} \\
2.87 \pm 0.3^{k}\end{array}$ \\
\hline
\end{tabular}

${ }^{a} \mathrm{MRCI}+\mathrm{Q}$, present work, ${ }^{b} \mathrm{MRCI}+\mathrm{Q},[23],{ }^{c} \mathrm{CCSDT}$, [25],

${ }^{d} \mathrm{SCF}+\mathrm{CI}$ and $\mathrm{MCSCF}+\mathrm{CI},[26],{ }^{e} \mathrm{CASSCF} / \mathrm{MRCI}[27]$ and ${ }^{f} \mathrm{RSPT} 2$ [27].

${ }^{g}$ RECP + MRDCI [22], ${ }^{h}$ Experiment, [28],

${ }^{i}$ Experiment, [29], ${ }^{j}$ Experiment, [30], ${ }^{k}$ Experiment, [31]. 
[26], a polarization propagator (SPPA) calculation [32], and Rayleigh Schrödinger Perturbation Theory to second order (RSPT2) $[27,33]$. From our ab initio work we extracted the molecular constants, $r_{e}$ the equilibrium bond length and the dissociation energy $D_{e}$ for these two states of the $\mathrm{SiO}$ molecule in order to compare with previous experimental and theoretical investigations. Table 1 shows our results and compares them to previous experiments and a selection of various theoretical methods. Our results are in excellent agreement with previous $\mathrm{MRCI}+\mathrm{Q}$ calculations of similar or slightly higher quality which have been extrapolated to the complete basis set limit (CBS). They are also in excellent agreement with the available experiments. The previous $\mathrm{MRCI}+\mathrm{Q}$ results that include the additional core-valence $(\mathrm{CV})$ and relativisitic effects incorporated through the Douglas Kroll method (DK) only give marginally better values for these molecular constants. We therefore have confidence in the molecular data for the PECs and TDMs to be used in our dynamical studies.

\section{Rate Constant}

The thermal rate constant (in $\mathrm{cm}^{3} \mathrm{~s}^{-1}$ ) at a given temperature $T$ to form a molecule by radiative association is given by

$$
k_{\Lambda \rightarrow \Lambda^{\prime}}=\left(\frac{8}{\mu_{r} \pi}\right)^{1 / 2}\left(\frac{1}{k_{B} T}\right)^{3 / 2} \int_{0}^{\infty} E \sigma_{\Lambda \rightarrow \Lambda^{\prime}}(E) e^{-E / k_{B} T} d E,
$$

where $\Lambda$ and $\Lambda^{\prime}$ are the initial and final projections of the electronic orbital angular momentum of the molecule on the internuclear axis, $\mu_{r}$ is the reduced mass of the $\mathrm{Si}+\mathrm{O}$ system, $k_{B}$ is Boltzmann's constant, and $E$ is the translational energy. The cross-section $\sigma_{\Lambda \rightarrow \Lambda^{\prime}}(E)$ may be calculated using semiclassical or quantal methods. The rate constant is often divided into a sum of two terms

$$
k_{\Lambda \rightarrow \Lambda^{\prime}}=k_{\Lambda \rightarrow \Lambda^{\prime}}^{\text {dir }}+k_{\Lambda \rightarrow \Lambda^{\prime}}^{r e s},
$$

where $k_{\Lambda \rightarrow \Lambda^{\prime}}^{\operatorname{dir}}$ is the non-resonant direct contribution which may be obtained from the semiclassical or quantum cross section, and $k_{\Lambda \rightarrow \Lambda^{\prime}}^{\text {res }}$ is the resonant contribution which must be obtained from a quantal method. We summarize these methods below.

\subsection{Semiclassical Method}

In the semiclassical approximation $[10,34,35]$, the cross-section for the radiative association process is given by

$$
\sigma_{\Lambda \rightarrow \Lambda^{\prime}}^{S C}(E)=2 \pi P_{\Lambda} \sqrt{\frac{2 \mu_{r}}{E}} \int_{0}^{+\infty} b d b \int_{R_{c}}^{\infty} \frac{\Gamma_{\Lambda \rightarrow \Lambda^{\prime}}(R) d R}{\sqrt{1-V_{\Lambda}(R) / E-b^{2} / R^{2}}}
$$

where

$$
\Gamma_{\Lambda \rightarrow \Lambda^{\prime}}(R)=2.03 \times 10^{-6}\left(\frac{2-\delta_{0, \Lambda+\Lambda^{\prime}}}{2-\delta_{0, \Lambda}}\right) \nu^{3}(R)|\mu(R)|^{2}
$$

is the radiative transition probability in $\mathrm{s}^{-1}$ at the given internuclear distance, $R_{c}$ is the classical turning point for the impact parameter $b, V_{\Lambda}(R)$ is the potential energy in the entrance channel, $\nu(R)$ is the photon energy in $\mathrm{cm}^{-1}$, and $\mu(R)$ is the transition moment in a.u. The statistical weight factor $P_{\Lambda}$ is given by

$$
P_{\Lambda}=\frac{\left(2 S_{m o l}+1\right)\left(2-\delta_{0, \Lambda}\right)}{\left(2 L_{S i}+1\right)\left(2 S_{S i}+1\right)\left(2 L_{O}+1\right)\left(2 S_{O}+1\right)},
$$


where $L_{S i}, S_{S i}, L_{O}, S_{O}$, are the electronic orbital and spin angular momenta of the silicon and oxygen atoms, and $S_{m o l}$ is the total spin of the molecular electronic state. For the $A^{1} \Pi \rightarrow X^{1} \Sigma^{+}$transition considered in this work, we obtain $P_{\Pi}=2 / 81$.

\subsection{Standard Quantum Theory}

The quantum mechanical cross-section $\sigma_{\Lambda \rightarrow \Lambda^{\prime}}^{Q M}(E)$ for the radiative association process can be calculated using perturbation theory for the radiative coupling (see Babb and Dalgarno [36], Gianturco and Gori [37] or Babb and Kirby [38]). The result is

$$
\sigma_{\Lambda \rightarrow \Lambda^{\prime}}^{Q M}(E)=\sum_{v^{\prime} j^{\prime}} \sum_{j} \frac{64}{3} \frac{\pi^{5} \hbar^{2}}{c^{3}} \frac{\nu^{3}}{2 \mu_{r} E} P_{\Lambda} S_{j j^{\prime}}\left|M_{\Lambda E j, \Lambda^{\prime} v^{\prime} j^{\prime}}\right|^{2},
$$

where the sum is over initial rotational $j$ and final vibrational $v^{\prime}$ and rotational $j^{\prime}$ quantum numbers. $S_{j, j^{\prime}}$ are the appropriate line strengths $[39,40]$ or Hönl-London factors [41], and $c$ is the speed of light. $M_{\Lambda E j, \Lambda^{\prime} v^{\prime} j^{\prime}}$ is given by the integral

$$
M_{\Lambda E j, \Lambda^{\prime} v^{\prime} j^{\prime}}=\int_{0}^{\infty} F_{\Lambda E j}(R) \mu(R) \Phi_{\Lambda^{\prime} v^{\prime} j^{\prime}}(R) d R .
$$

The wavefunction $\Phi_{\Lambda^{\prime} v^{\prime} j^{\prime}}(R)$ is a unit-normalized bound state eigenfunction of the final electronic state, and $F_{\Lambda E j}(R)$ is an energy-normalized continuum wavefunction of the initial electronic state. These wavefunctions may be computed from their respective Schrödinger equations using a grid-based numerical approach $[6,7,36,42,43]$. Complicated resonance structures generally make it difficult to calculate the rate coefficient (2) using numerical integration [12,42]. Furthermore, the majority of these resonances are sufficiently narrow that the probability of tunneling is negligible compared to the radiative decay probability. Consequently, perturbation theory for extremely narrow resonances can yield opacities which are larger than unity $[7,12,42]$. An optical potential approach which includes radiative broadening may be used to handle the narrow resonances in order to ensure unitarity [13,42]. This approach may also be used to derive a formula for the resonance contribution $[7,11-13,44]$

$$
k_{\Lambda \rightarrow \Lambda^{\prime}}^{r e s}=\sum_{q} K_{q}^{e q} \frac{\Gamma_{q}^{t u n} \Gamma_{q}^{r a d}}{\Gamma_{q}^{t u n}+\Gamma_{q}^{r a d}},
$$

where $q \equiv\left(v_{q}, j_{q}\right)$ designates the vibrational and rotational quantum numbers for a quasibound state, $\Gamma_{q}^{\text {tun }}$ and $\Gamma_{q}^{\text {rad }}$ are the respective tunneling and radiative decay widths, and $K_{q}^{e q}$ is the equilibrium constant for the quasibound state given by

$$
K_{q}^{e q}=\frac{\left(2 j_{q}+1\right) P_{\Lambda} \exp \left(-E_{q} / k_{B} T\right)}{Q_{T}} .
$$

Here $Q_{T}$ is the translational partition function, and $E_{q}$ is the energy of the quasibound state. The key step in the derivation of (9) is the replacement of the tunneling width $\Gamma_{q}^{\text {tun }}$ in the Lorentzian of a Breit-Wigner resonance by the total decay width $\Gamma_{q}^{\text {tun }}+\Gamma_{q}^{\text {rad }}$. Equation (9) provides an equilibrium population of quasibound states when the tunneling probability is large compared to the radiative decay width. For extremely long-lived quasibound states such that $\Gamma_{q}^{t u n}<<\Gamma_{q}^{r a d}$, however, the ratio in equation (9) goes to zero. This implies that all narrow resonances make negligible contributions to the formation rate. The standard quantum theory result is obtained when the resonance contribution (9) is added to the direct non-resonance contribution computed from equation (7). The Sturmian approach [45] described in the next section provides an alternative method for calculating the standard quantum theory rate constant if long-lived quasibound states are eliminated as in equation (9). 


\subsection{Quantum Kinetic Theory}

In this section, we follow the approach described by Forrey [14] and define the radiative association rate coefficient from the steady-state solution of a self-consistent master equation. The formulation uses the bound and unbound energy eigenstates of a Sturmian representation to form a complete basis set for both the dynamics and kinetics. All possible transitions are included in the master equation which allows the extremely long-lived quasibound states to be populated through bound-unbound and unbound-unbound interactions. The result is a phenomenological rate constant that includes both direct and indirect (inverse predissociation) processes given by

$$
k_{\Lambda \rightarrow \Lambda^{\prime}}=\sum_{b, u} K_{u}^{e q}\left(1+\delta_{u}\right) \Gamma_{u \rightarrow b}^{r a d}
$$

where $b \equiv\left(v_{b}, j_{b}\right)$ and $u \equiv\left(v_{u}, j_{u}\right)$ designate vibrational and rotational quantum numbers for bound and unbound states, respectively. $\Gamma_{u \rightarrow b}^{r a d}$ is the radiative transition probability, and $K_{u}^{e q}$ is the equilibrium constant for the unbound state. The parameter $\delta_{u}$ is a non-equilibrium concentration defect which depends on tunneling lifetimes and may be computed as a function of the matter and radiation temperatures. For LTE, it was shown [14] that the concentration defects are identically zero. The resulting expression is then equivalent to equation (2) using the cross section

$$
\sigma_{\Lambda \rightarrow \Lambda^{\prime}}(E)=\frac{\pi^{2} \hbar^{3}}{\mu_{r} E} P_{\Lambda} \sum_{b, u}\left(2 j_{u}+1\right) \Gamma_{u \rightarrow b}^{r a d} \delta\left(E-E_{u}\right)
$$

where the delta function is due to the Möller operator which transforms a free Sturmian eigenstate into an interacting unbound state with the same energy. For comparison, we note that standard quantum theory generally uses equations (2) and (7) assuming a Lorentzian line shape

$$
\left|M_{\Lambda E j, \Lambda^{\prime} v^{\prime} j^{\prime}}\right|^{2} \equiv \frac{\Gamma_{q}^{\text {tun }} / 2 \pi}{\left(E-E_{q}\right)^{2}+\left(\Gamma_{q}^{\text {tun }} / 2\right)^{2}}\left|M_{q \rightarrow b}\right|^{2}
$$

to obtain an equation which appears similar to equation (11) for the unbound subspace consisting of only the quasibound states. Radiative broadening (ie. $\Gamma_{q}^{t u n} \rightarrow \Gamma_{q}^{t u n}+\Gamma_{q}^{r a d}$ in the denominator of the Lorentzian) then yields the resonance formula (9).

There are two key differences between the quantum kinetic result (11) and the resonance formula (9): (i) equation (11) includes the non-resonant background contribution, and (ii) contributions from long-lived quasibound states are not eliminated from equation (11). This second difference is mathematically equivalent to the neglect of radiative broadening in the Lorentzian used to derive equation (9). However, there is no breakdown of perturbation theory or violation of unitarity in the result derived from quantum kinetic theory [14]. It should be noted that LTE in the present context means that the matter and radiation temperatures are the same. When this is the case, the steady-state solution of the Sturmian master equation is a Boltzmann distribution for all unbound states, independent of density and tunneling lifetime. Therefore, all unbound states, including extremely long-lived quasibound states, should be included in the formation rate constant (11). For comparison, the resonance formula (9), which excludes contributions from long-lived quasibound states, may be obtained from quantum kinetic theory as an approximate steady-state solution of the master equation for a low density gas at zero radiation temperature [14]. 


\section{Results and Discussion}

In this section, we present results from the semiclassical, standard quantum, and quantum kinetic theories. For the standard quantum and quantum kinetic results, we employed the Sturmian method $[14,45]$ to compute the bound and unbound wavefunctions needed to evaluate the matrix elements (8). Quasibound states which have negligible tunneling probability compared to their radiative decay probability were not included in the standard quantum result in accordance with equation (9). The long-lived quasibound states, however, were retained in the quantum kinetic theory formula (11) which is evaluated in the present work for LTE conditions. The radiative transition probability includes spontaneous and stimulated emission and is given by

$$
\Gamma_{u \rightarrow b}^{r a d}=\frac{A_{u \rightarrow b}}{1-e^{-\left(E_{u}-E_{b}\right) / k_{B} T}}
$$

where

$$
A_{u \rightarrow b}=\frac{4}{3 c^{3}}\left(E_{u}-E_{b}\right)^{3} S_{j_{u} j_{b}}\left|M_{u \rightarrow b}\right|^{2}
$$

is the Einstein A-coefficient connecting the bound and unbound eigenstates which were computed by separately diagonalizing the Hamiltonian matrices for the $\mathrm{X}^{1} \Sigma^{+}$ and $\mathrm{A}^{1} \Pi$ electronic states in an $L^{2}$ Sturmian representation [45] consisting of 500 Laguerre polynomial basis functions per partial wave. As discussed previously [45], it is sufficient to use unit-normalized positive energy eigenstates directly because the usual energy normalization exactly cancels the equivalent quadrature weights which are needed to express the integration over unbound states as a discrete sum. This allows the rate constant given in equation (11) to be easily computed by multiplying equation (14) by the equilibrium constant and summing over the bound and unbound states.

Fig 3 shows the relative importance of the resonant and non-resonant contributions for a few partial waves. In the figure, the cumulative radiative width

$$
\Gamma_{u}=\sum_{b} \Gamma_{u \rightarrow b}^{r a d}
$$

is plotted as a function of the unbound energy $E_{u}$ for zero temperature. The figure shows no resonances for $j_{u}=0$ and an increasing curve due to the increasing energy gap between the bound and unbound states. The increase stops abruptly at $E_{u} \approx 0.2$ a.u. due to the fall-off of Franck-Condon factors before further increasing as the energy gap continues to widen. A similar step-like structure is found near 0.2 a.u. for the higher partial waves, however, there are very narrow resonances that may be seen at lower energies. The resonances eventually vanish for large angular momentum (e.g. $j_{u}=300$ ). In our calculations, we found that the last bound state for the ground state $\left(\mathrm{X}^{1} \Sigma^{+}\right)$potential occurs for $j_{b}=329$, so we included all 500 bound and unbound vibrational states for $j_{u}=0-330$.

Fig 4 shows the rate coefficient for radiative association of $\operatorname{Si}\left({ }^{3} P\right)$ and $\mathrm{O}\left({ }^{3} P\right)$ via the $\mathrm{A}^{1} \Pi$ molecular state. The present results are compared against the previous semiclassical result of Andreazza and co-workers [9]. The standard quantum result was computed from equation (11) by removing all quasibound states that have negligible tunneling probability compared to their radiative decay width. This condition is met when the sum over Franck-Condon factors is greater than 1.99 for the $j \pm 1$ transitions. Fig 4 shows that the standard quantum curve is in good agreement with the present semiclassical curve. However, both of these curves are significantly 


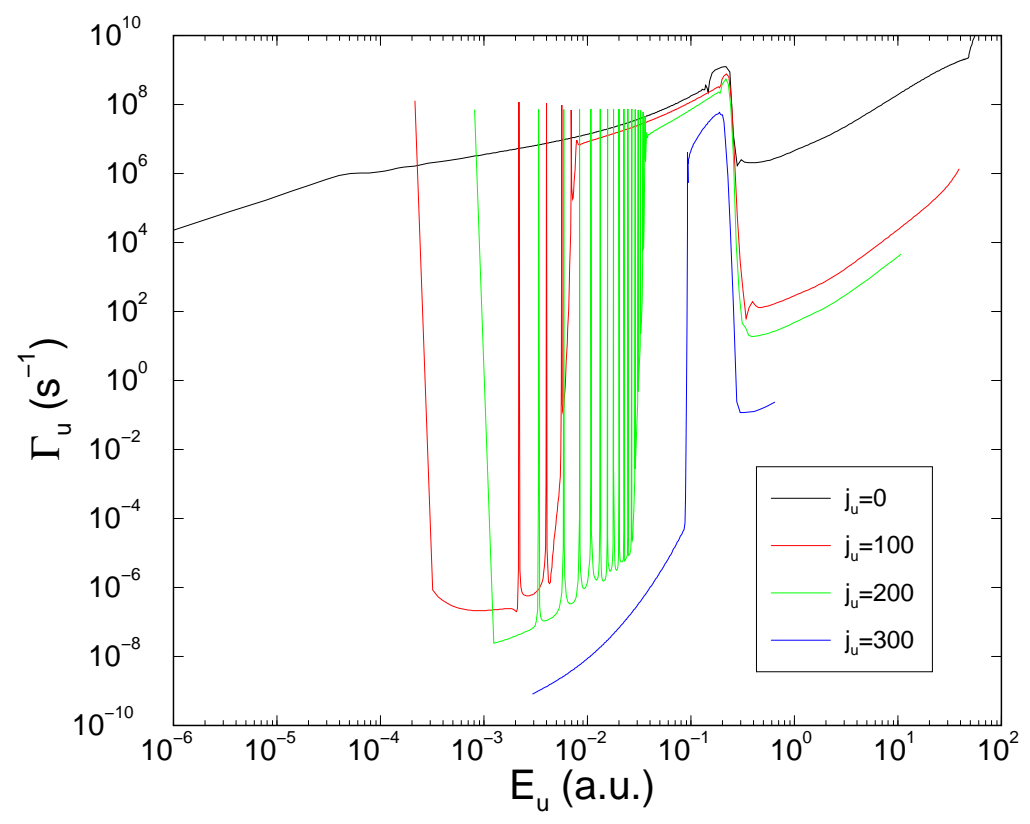

Figure 3. (Colour online) The cumulative radiative width plotted as a function of the unbound energy eigenvalues $E_{u}$ for $j_{u}=0,100,200$, and 300 . The prominant narrow resonant features are clearly visible in the $j_{u}=100$ and 200 values. The cross section can be obtained from the plotted radiative width via equation (12).

smaller than the previous semiclassical calculation $\S$. This difference is presumably due to the improved molecular data used in the present work. When the narrow resonances are included, the quantum kinetic theory curve shows a difference which is even more substantial. The rate coefficient increases with decreasing temperature and is about 100 times larger than the standard quantum result which excludes all of the narrow resonances. It should be noted that the standard quantum curve does include broad resonances, which is the cause of the slight upturn in the curve at low temperature. Stimulated emission is also included in the quantum calculations [46]. When stimulated association is excluded (e.g. dashed blue curve), the quantum results merge smoothly with the semiclassical calculation at high temperatures.

\section{Conclusions}

We have computed high accuracy $\mathrm{SiO}$ molecular structure data for the purpose of studying radiative association in $\mathrm{Si}$ and $\mathrm{O}$ atom collisions. Semiclassical and quantum mechanical rate coefficients are reported, and the impact of very narrow resonances is analyzed using a quantum kinetic theory. The present semiclassical and standard quantum theory results agree with each other, however, they are significantly smaller in magnitude than a previous semiclassical result. The quantum kinetic theory includes the extremely narrow resonances and yields a result which is orders of magnitude larger than the standard quantum result and has a different temperature dependence.

$\S$ The rate coefficients listed in Table I of [9] include contributions from $\mathrm{E}^{1} \Sigma^{+}$, but they are typically much smaller in magnitude rising to only $41 \%$ of the total rate at $14,700 \mathrm{~K}$. 


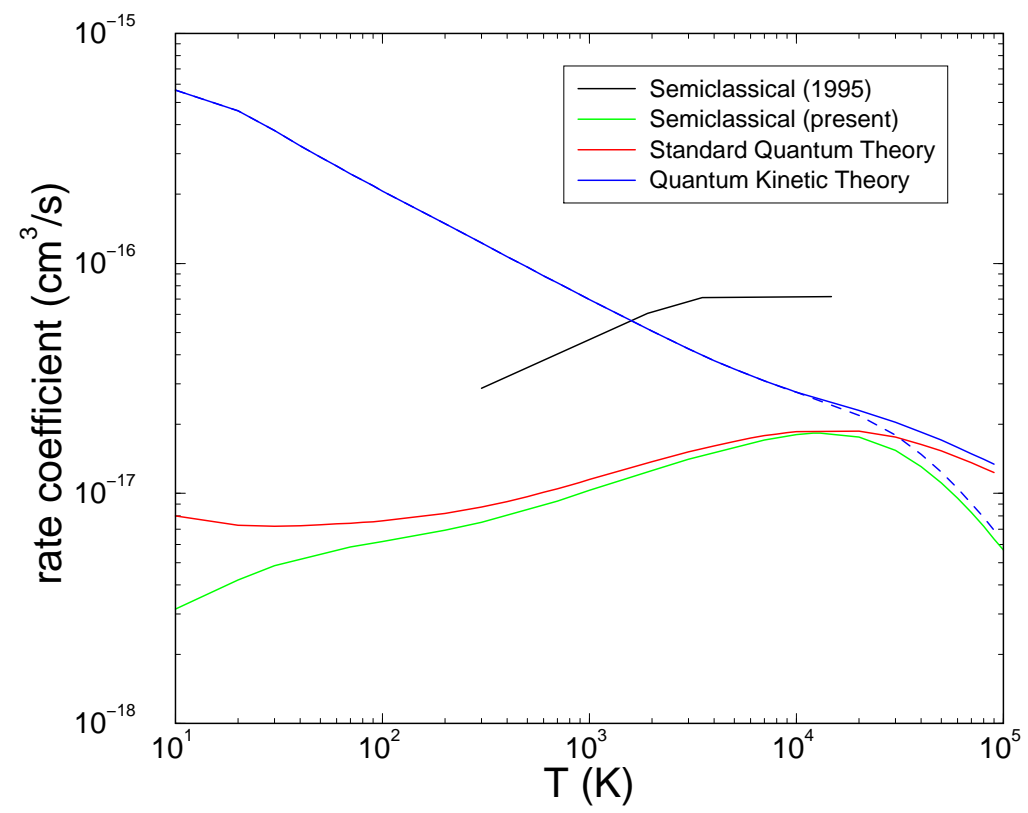

Figure 4. (colour online) Rate coefficient for radiative association of $\operatorname{Si}\left({ }^{3} P\right)$ and $\mathrm{O}\left({ }^{3} P\right)$ via the $\mathrm{A}^{1} \Pi$ molecular curve. Present results include semiclassical (green), standard quantum (red), and quantum kinetic (blue) curves. Also shown (black) is the previous semiclassical result of Andreazza et al [9]. Note that the quantum calculations include stimulated emission. The semiclassical result and the dashed blue quantum kinetic curve include spontaneous emission only.

The justification for including extremely narrow resonances comes from the self-consistent master equation which requires that all quasibound states are fully populated at LTE. It was further shown $[14,47]$ that non-LTE conditions may reduce the resonant contribution or possibly lead to resonant enhancements which are even larger than those reported here. This raises an important question about which rate constant should be used in an astrochemical model. The vast majority of astrochemical network models are not state-resolved, and the molecules are assumed to be in their ground state. Furthermore, the matter and radiation temperatures are rarely the same in typical low density ISM environments. The "standard quantum theory" result corresponds to zero radiation temperature at low density and should be used for modelling such environments. For molecular clouds which receive light from bright background stars or are sufficiently dense that quasibound states may be populated through inelastic collisions, the LTE result would be more appropriate. A more detailed study of the present system which includes a full dependence of the resonant contribution on both the matter and radiation temperatures is clearly warranted.

In order to compare several collisional theories, we calculated the rate coefficient for the formation of $\mathrm{SiO}$ via radiative association along the $\mathrm{A}^{1} \Pi$ electronic state. For astrophysical applications, a complete treatment of $\mathrm{SiO}$ formation from radiative collisions of $\mathrm{Si}$ and $\mathrm{O}$ must account for approach along the other electronic states. The increasing formation rate with decreasing temperature due to the resonances may have important implications for astrochemical models of cold molecular clouds. 


\section{Acknowledgments}

RCF acknowledges support from NSF Grant Nos. PHY-1203228 and PHY-1503615. PCS acknowledges support from NASA grant NNX15AI61G. BMMcL acknowledges support from NSF through a grant to ITAMP at the Harvard-Smithsonian Center for Astrophysics under the visitors program, and Queen's University Belfast for the award of a visiting research fellowship (VRF). The electronic structure work was carried out at the National Energy Research Scientific Computing Center in Oakland, CA, USA and at the High Performance Computing Center Stuttgart (HLRS) of the University of Stuttgart, Stuttgart, Germany.

\section{References}

[1] Lepp S, Dalgarno A and McCray R 1990 Astrophys. J 358262

[2] Liu W, Dalgarno A and Lepp S 1992 Astrophys. J 396679

[3] Liu $\mathrm{W}$ and Dalgarno A 1996 ApJ $471 \quad 480$ ISSN $0004-637 \mathrm{X}$ URL http://stacks.iop.org/0004-637X/471/i=1/a=480

[4] Cherchneff I and Sarangi A 2011 Proc. Int. Astron. Union 7 S280 228

[5] Dalgarno A, Du M L and You J H 1990 Astrophys. J 349675

[6] Franz J, Gustafsson M and Nyman G 2011 Mon. Not. Roy. Astr. Soc. 4143547 URL http://dx.doi.org/10.1111/j.1365-2966.2011.18654x/

[7] Antipov S V, Gustafsson M and Nyman G 2013 Mon. Not. Roy. Astr. Soc. 430946 URL http://dx.doi.org/10.1093/mnras/sts615/

[8] Marassi S, Schneider R, Limongi M, Chieffi A, Bocchio M and Bianchi S 2015 arXiv URL http://arxiv.org/abs/1509.08923/

[9] Andreazza C M, Marinho E P and Singh P D 1995 Astrophys. J 451889

[10] Bates D R 1951 Mon. Not. Roy. Astr. Soc. 111303

[11] Bain R A and Bardsley J N 1972 J. Phys. B: At. Mol. Phys. 5277 URL http://iopscience.iop.org/0022-3700/5/2/024/

[12] Bennett O J, Dickinson A S, Leininger T and Gadéa X 2003 Mon. Not. Roy. Astr. Soc. 341 361 [erratum: 384, 1743] URL http=//dx.doi.org/10.1046/j.1365-8711.2003.06422.x/

[13] Mrugala F, Spirko V, and Kraemer W P 2003 J. Chem. Phys. 11810547

[14] Forrey R C 2015 J. Chem. Phys. 143024101 URL http://dx.doi.org/10.1053/1.4926325/

[15] Helgaker T, Jørgesen P and Oslen J 2000 Molecular Electronic-Structure Theory (New York, USA: Wiley)

[16] Kendall R A, Dunning T H and Harrison R J 1992 J. Chem. Phys. 96 6796 URL http://dx.doi.org/10.1063/1.462569/

[17] Woon D E and Dunning T H 1993 J. Chem. Phys. 98 1358 URL http://dx.doi.org/10.1063/1.464303/

[18] Mourik T V, Wilson A K and Dunning T H 1999 Mol. Phys. $96 \quad 529$ URL http://dx.doi.org/10.1016/0009-2614(85)80025-7/

[19] Werner H J, Knowles P J, Manby F R, Schütz M, et al MOLPRO 2012 URL http://www.molpro.net

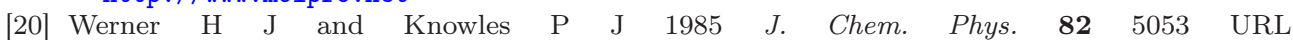
http://dx.doi.org/10.1063/1.448627

[21] Werner H J and Knowles P J 1985 Chem. Phys. Lett. 115259 URL http://dx.doi.org/10.1016/0009-2614(85)80025-7/

[22] Chattopadhyaya S, Chattopadhyay A and Das K K 2003 J. Phys. Chem. A 107148

[23] Shi D, Li W, Sun J and Zhu Z 2012 Spectrochimica Acta Part A 87 96 URL http://dx.doi.org/10.10161/j.saa.2011.11.017/

[24] Barton E J, Yurchenko S N and Tennyson J 2013 Mon. Not. Roy. Astr. Soc. 4341469 URL http://dx.doi.org/10.1093/mnras/stt1105/

[25] Drira I, Huré J M, Spielfiedel A, Feautrier N and Roueff E 1997 Astron. Astrophys. 319720

[26] Langhoff S R and Arnold J $\mathrm{O} \quad 1979 \quad$ J. Chem. Phys. 70 852 URL http://dx.doi.org/10.1063/1.437491/

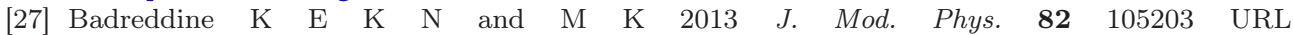
http://dx.doi.org/10.4236/jmp. 2013.41014/ 
[28] Huber K P and Herzberg G 1979 Molecular Spectra and Molecular Structure IV: Constants of Diatomic Molecules (Princeton, New Jersey, USA: Van Nostrand-Reinhold)

[29] Hildenbrand D L 1972 H. Temp. Sci. 4244

[30] Brewer K and Rosen Blatt 1969 Advances in High-Temperature Chemistry: Volume 2 (New York, USA: Academic)

[31] Gaydon A G 1968 Dissociation energies and Spectra of Diatomic Molecules (London, UK: Chamman and Hall)

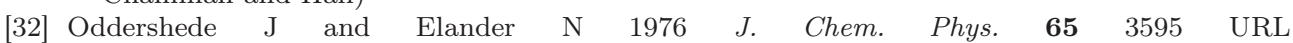
http://dx.doi.org/10.1088/0004-637X/770/1/33/

[33] Charropadhyaya S, Chattopadhyay A and Das K K 2003 J. Phys. Chem. A 107148 URL http://dx.doi.org/10.1021/jp021845v/

[34] Zygelman B and Dalgarno A 1988 Phys. Rev. A 381877

[35] Zygelman B and Dalgarno A 1990 Astrophys. J 365239

[36] Babb J F and Dalgarno A 1995 Phys. Rev. A $51 \quad 3021$ URL http://dx.doi.org/10.1103/PhysRevA.54.4073/

[37] Gianturco F A and Gori Giorgi P 1996 Phys. Rev. A $54 \quad 4073$ URL http://dx.doi.org/10.1103/PhysRevA.54.4073/

[38] Babb J F and Kirby K P 1998 Molecule Formation in Dust-poor Environments Molecular Astrophysics of Stars and Galaxies ed T W Hartquist and D A Williams (Oxford, UK: Clarendon Press) p 11

[39] Cowan R D 1981 The Theory of Atomic Structure and Spectra (Berkeley, California, USA: University of California Press)

[40] Curtis L J 2003 Atomic Structure and Lifetimes: A conceptual Approach (Cambridge, UK: Cambridge University Press)

[41] Watson J K G 2008 J. Molec. $\quad$ Spectrosc. 25350 URL http://dx.doi.org/10.1016/j.jms.2008.04.014/

[42] Gutafsson M, Antipov S V, Franz J and Nyman G 2012 J. Chem. Phys. 137104301 URL http $=/ /$ dx. doi. org $/ 10.1063 / 1.4750029 /$

[43] Antipov S V, Sjölander T, Nyman G and Gustafsson M 2009 J. Chem. Phys. 131074302 URL http=//dx . doi .org/10.1063/1.3196178/

[44] Carrington T 1972 J. Chem. Phys. 572033 URL http://dx.doi.org/10.1063/1.1678527/

[45] Forrey R C 2013 Phys. Rev. A 88052709 URL http://dx.doi.org/10.1103/PhysRevA. 88.052709/

[46] Stancil P C and Dalgarno A 1997 Astrophys. J 479543

[47] Forrey R C submitted 2016 J. Phys. B 